\title{
WHITEHEAD THEOREMS IN PROPER HOMOTOPY THEORY
}

\author{
BY DAVID A. EDWARDS AND HAROLD M. HASTINGS
}

Communicated by P. T. Church, August 13, 1975

Following Chapman [3] we define a continuous map $f: X \rightarrow Y$ to be proper iff for each compactum $B \subset Y$ there exists a compactum $A \subset X$ such that $f(X \backslash A) \cap B=\varnothing$. (This is just a reformulation of the usual notion of a proper map.) Then maps $f, g: X \rightarrow Y$ are said to be weakly properly homotopic iff for each compactum $B \subset Y$ there exists a compactum $A \subset X$ and a homotopy (dependent on $B) F=\left\{F_{t}\right\}: X \times I \rightarrow Y$ (where $I=[0,1]$ ) such that $F_{0}=f$, $F_{1}=g$, and $F((X \backslash A) \times I) \cap B=\varnothing$. If, in fact, there exists a proper map $F: X \times I \rightarrow Y$ which satisfies $F_{0}=f$ and $F_{1}=g$, then we say that $f$ and $g$ are properly homotopic. The notions of weak proper homotopy equivalence and proper homotopy equivalence are now defined in the obvious way.

In [7, pp. 489-491] Siebenmann obtained various convenient criteria for a proper map of locally finite simplicial complexes to be a proper homotopy equivalence. Siebenmann's proof seemed to require a finite dimensional assumption. Later, E. Brown [2, p. 34], and Farrell, Taylor and Wagoner [6] claimed to be able to remove the finite dimensional assumption. In [4] we give an example, using an interesting map of J. F. Adams [1], which shows that the finite dimensional assumption is necessary. On the positive side, we prove in [4] the following useful (see [5]) Whitehead type theorem.

THEOREM. Let $f: X \rightarrow Y$ be a proper map of locally finite simplicial complexes such that $f$ is a weak proper homotopy equivalence. Then $f$ is weakly properly homotopic to a proper homotopy equivalence.

\section{REFERENCES}

1. J. F. Adams, On the groups $J(X)$. IV, Topology 5 (1966), 21-71; correction, ibid. 7 (1968), 331. MR 33 \#6628; 37 \#5874.

2. E. M. Brown, On the proper homotopy type of simplicial complexes, Topology Conf., 1973, edited by R. F. Dickman, Jr. and P. Fletcher, Lecture Notes in Math., vol. 375, Springer-Verlag, Berlin and New York, 1974.

3. T. A. Chapman, On some applications of infinite-dimensional manifolds to the theory of shape (to appear).

4. D. Edwards and H. Hastings, Whitehead theorems in proper homotopy theory (to appear).

5. D. Edwards, H. Hastings and J. West, Group actions on infinite dimensional manifolds (mimeographed notes).

AMS (MOS) subject classifications (1970). Primary 55D10; Secondary 55B99. theorems.

Key words and phrases. Pro-homotopy theory, proper homotopy theory, Whitehead 
6. F. T. Farrell, L. R. Taylor and J. B. Wagoner, The Whitehead theorem in the proper category, Compositio Math. 27 (1973), 1-23. MR 48 \#12545.

7. L. C. Siebenmann, Infinite simple homotopy types, Nederl. Akad. Wetensch. Proc. Ser. A 73 = Indag. Math. 32 (1970), 479-495. MR 44 \#4746.

DEPARTMENT OF MATHEMATICS, STATE UNIVERSITY OF NEW YORK AT BINGHAMTON, BINGHAMTON, NEW YORK 13901

DEPARTMENT OF MATHEMATICS, HOFSTRA UNIVERSITY, HEMPSTEAD, NEW YORK 11550 